\title{
Oral fluoropyrimidine-induced severe hyperlipidemia
}

\author{
Bulent Yildiz ${ }^{\mathrm{a}}$, Halil Kavgaci ${ }^{\mathrm{a}}$ Evren Fidan ${ }^{\mathrm{a}}$,Erdal Gungor ${ }^{\mathrm{b}}$, Halil O. Ersoz $^{\mathrm{c}}$, Feyyaz Ozdemir ${ }^{\mathrm{a}}$, Fazil Aydin ${ }^{\mathrm{a}}$ \\ Department of Medical Oncology ${ }^{a}$,Department of Internal Medicine ${ }^{b}$, Department of Endocrinology ${ }^{c}$, \\ Faculty of Medicine, Karadeniz Technical University, Trabzon 61080, Turkey
}

\begin{abstract}
Background: Severe hyperlipidemia secondary to capecitabine, an oral fluoropyrimidine, is a very rare condition. There are no reported cases of hyperlipidemia associated with Uracil/tegafur (UFT).

Objective: Report UFT-induced severe hyperlipidemia.

Method: A 71-year-old male patient with metastatic colorectal cancer receiving capecitabine treatment was hospitalized at the end of the eighth cycle with the complaint of fatigue. Capecitabine treatment was discontinued in the patient in whom severe hyperlipidemia was detected together with disease progression. Gemphibrozile 1200 mg/day was initiated; patient's triglyceride level and serum cholesterol decreased from 1768 to $149 \mathrm{mg} / \mathrm{dL}$ and from 497 to $99 \mathrm{mg} / \mathrm{dL}$, respectively, five weeks later. The patient started to receive UFT chemotherapy and after the second cycle, he presented to our hospital again with the complaints of fatigue, headache, and yellow vision. The investigations revealed a serum triglyceride level of $4115 \mathrm{mg} / \mathrm{dL}$ and a cholesterol level of $734 \mathrm{mg} / \mathrm{dL}$. Results: UFT chemotherapy was discontinued and lipopheresis was administered for three consecutive days, and gemphibrozile was initiated again at a dose of $1200 \mathrm{mg} /$ day. The clinical presentation might be due to oral fluoropyrimidine. Three weeks later, serum cholesterol and triglyceride levels decreased to 106 and $403 \mathrm{mg} / \mathrm{dL}$, respectively.

Conclusion: This case is a unique case of hyperlipidemia secondary to UFT. Monitoring of lipid levels, when using Fluoropyrimidine, as well as hemograms, liver and renal functions would be appropriate.
\end{abstract}

Keywords: Adverse event, capecitabine, hypertriglyceridemia, oral fluoropyrimidine, UFT ${ }^{\circledR}$ drug

Oral fluoropyrimidines are agents that have been shown to be effective in the treatment of metastatic rectal cancer $[1,2]$. Uracil/tegafur $\left(\mathrm{UFT}^{\circledR}\right)$ is a dihydropyrimidine dehydrogenase inhibitor that consists of tegafur and uracil at a molar rate of 1:4 [1]. Capecitabine exerts its efficacy by transforming into 5-fluorouracil (5-FU) in the tumor cell [2]. Although they are well-tolerated agents, their most common side effects include palmar-plantar erythrodysesthesia (hand-foot syndrome), diarrhea, and stomatitis. In the literature, the incidence of grade 3-4 hypertriglyceridemia (5 to 10 fold of the normal limit or $>10$ fold increase) was reported at a rate of $0.1-1 \%$ [3].

In this report, we discussed a case of metastatic rectal cancer with a severe increase detected in serum

Correspondence to: Bulent YILDIZ, MD. Department of Medical Oncology, Faculty of Medicine, Karadeniz Technical University, Trabzon 61080, Turkey. E-mail: drbulentyildiz@ hotmail.com triglyceride and cholesterol levels with both capecitabine and UFT use.

\section{Case report}

The 71-year-old male patient underwent low anterior resection due to rectal cancer in March 2005. He was administered adjuvant 5-FU-folinic acid and concomitant radiotherapy under the diagnosis of stage IIIA rectum cancer. Upon detection of liver and bone metastases in September 2006, capecitabine (1250 mg/m² twice a day for 14 days every 21 days) and oxaliplatin (130 $\mathrm{mg} / \mathrm{m}^{2}$ every 21 days) chemotherapy was initiated. Patient's medical history included 20 years of type II diabetes and hyperlipidemia, and coronary bypass operation performed five years ago. Pre-treatment body mass index, serum glucose, cholesterol (normal range: 120200), triglyceride (TG) (normal range: 50-150), highdensity lipoprotein (HDL) (normal range: 35-70) and low-density lipoprotein (LDL) (normal range: 65-175) were $26,161,229,120,47$, and $158 \mathrm{mg} / \mathrm{dL}$, respectively. 
After four cycles of chemotherapy in the patient with grade I palmar-plantar erythrodysesthesia, partial remission was achieved. Oxaliplatin was discontinued and the dose of capecitabine was decreased $\left(1000 \mathrm{mg} / \mathrm{m}^{2}\right)$ and the treatment was continued for another four cycles. In May 2007 (after eight cycles of treatment), the patient presented with complaints of grade III palmar-plantar erythrodysesthesia and fatigue. Laboratory investigations revealed severe hyperlipidemia (serum cholesterol, TG, HDL, and LDL were 497, 1768, 70, and $39 \mathrm{mg} / \mathrm{dL}$, respectively). Potential risk factors including changes in dietary regimen, weight gain, alcohol use, uncontrolled diabetes and hypothyroidism were excluded. Treatment was discontinued, considering that acute hypertriglyceridemia was secondary to capecitabine treatment. Gemfibrozil 1200 mg/day was initiated together with a low fat diet.

After five weeks, serum lipid parameters regressed to normal limits and the patient was started on UFT ( $300 \mathrm{mg} / \mathrm{m}^{2} /$ day, in two divided doses given on days 1-14 every three weeks) chemotherapy. After two cycles of chemotherapy, the patient presented again to our hospital with complaints of fatigue, lack of appetite, abdominal pain, headache, and yellow vision. Serum cholesterol and TG levels were 734 and $4115 \mathrm{mg} / \mathrm{dL}$, respectively.

Risk factors that may result in hypertriglyceridemia were re-evaluated and no reason other than UFT could be detected. The patient whose treatment was discontinued underwent lipopheresis for three consecutive days. After lipopheresis, serum TG level was determined to be $1953 \mathrm{mg} / \mathrm{dL}$. Gemfibrozil 1200 $\mathrm{mg} /$ day was initiated again and three weeks later, serum cholesterol and TG levels declined to 106 and $403 \mathrm{mg} / \mathrm{dL}$, respectively. The patient followed up with response of stable disease was detected to have progression in June 2009 and FOLFIRI-cetuximab treatment was initiated. In May 2009, the patient's serum TG and cholesterol level was $128 \mathrm{mg} / \mathrm{dL}$ and $219 \mathrm{mg} / \mathrm{dL}$, respectively.

In Table 1 are shown information regarding our patient and other five patients that were available in the literature.

\section{Discussion}

There is no reported case of hypertriglyceridemia secondary to UFT use in the literature, but there are 10 cases of hypertriglyceridemia secondary to capecitabine [3-6]. Five of these cases were completely described, and the remaining was detected by monitoring serum lipid parameters of other patients upon incidental detection of hypertriglyceridemia in a trial investigation of the efficacy and side-effects of capecitabine [4].

Information regarding six patients with previously published data as well as our patient is presented in Table 1. Four patients were receiving capecitabine at a dose of $2500 \mathrm{mg} / \mathrm{m}^{2} /$ day, and two started treatment at a dose of $2000 \mathrm{mg} / \mathrm{m}^{2} /$ day. In two patients, capecitabine dose was decreased by $25 \%$ after the increase in serum TG levels and the treatment was continued at this dose. However, TG levels increased again in both patients despite the dose reduction. Treatment had to be interrupted in three patients due to the increase in TG levels. Although serum lipid levels became normal after five-week interruption of treatment, a reduction of approximately 1.5 fold of the normal was detected in the other two cases. Both patients were receiving antihyperlipidemic treatment. Only in the case with colorectal cancer, serum TG level increased by approximately nine folds after two cycles, and $20 \mathrm{mg} /$ day atorvastatin was added to treatment, and the therapy was continued for further four cycles. The patient completed his treatment with a TG level that is approximately 1.5 fold higher than the normal without requirement for treatment interruption.

Considering all the cases in the literature, there is no other case detected to have hypertriglyceridemia with both capecitabine and UFT use. In the literature, the highest serum TG level detected to date was 3090 $\mathrm{mg} / \mathrm{dL}$ (15.5 fold of the upper limit), but in our patient, the serum TG level was detected as $4115 \mathrm{mg} / \mathrm{dL}$ (27.4 fold of the upper limit) following two cycles of UFT.

Up to now, no hypertriglyceridemia secondary to 5-FU use have been reported. However, Stathopoulos et al. [7] detected a fall in TG levels only in rabbits while a decrease in serum cholesterol level was observed in human and animal subjects given 5-FU. This suggests that there may be a relation between 5-FU and the lipid metabolism. There are no cases of hypertriglyceridemia associated with UFT use in the literature.

The etiology of hypertriglyceridemia induced by oral fluoropyrimidines is unknown yet. Certainly, these agents (or their intermediate metabolites) might occur during transformation to 5-FU may be involved. However, considering its observation in only $0.1-1 \%$ of the patients, differences in drug metabolism and 
genetic predisposition in lipid metabolism (i.e. lipoprotein lipase deficiency) might be involved in development of hypertriglyceridemia.

Another reason why hypertriglyceridemia secondary to oral fluoropyrimidines is serum lipid profile is not routinely monitored in these patients. Likewise, Koutras et al. [5] added regular lipid level monitoring to their study protocol upon incidental detection of high serum lipid levels in a patient, and TG levels were detected to be three to four fold higher than the normal in five of the 12 patients. Therefore, we believe that regular monitoring of lipid levels would be appropriate in patients who are using oral fluoropyrimidine.

The authors have no conflict of interest to declare.

\section{References}

1. Carmichael J, Popiela T, Radstone D, Falk S, Borner M, Oza A, et al. Randomized comparative study of tegafur/uracil and oral leucovorin versus parenteral fluorouracil and leucovorin in patients with previously untreated metastatic colorectal cancer. J Clin Oncol. 2002; 20:3617-27.

2. Van Cutsem E, Twelves C, Cassidy J, Allman D,
Bajetta E, Boyer M, et al. Oral capecitabine compared with intravenous fluorouracil plus leucovorin in patients with metastatic colorectal cancer: results of a large phase III study. J Clin Oncol. 2001; 19:4097-106.

3. Bar-Sela G, Haim N. Uncontrolled hypertriglyceridemia induced by capecitabine: case report and review of the literature. Cancer Chemother Pharmacol. 2009; 63: 779-82.

4. Stathopoulos GP, Koutantos J, Lazaki H, Rigatos SK, Stathopoulos J, Deliconstantinos G. The marked words were removed. Capecitabine (Xeloda) as monotherapy in advanced breast and colorectal cancer: effectiveness and side-effects. Anticancer Res. 2007; 27: 1653-6.

5. Koutras AK, Habeos IG, Vagenakis AG, Kalofonos HP. Capecitabine-induced hypertriglyceridemia: a report of two cases. Anticancer Res. 2006; 26: 2249-51.

6. Kurt M, Babaoglu MO, Yasar U, Shorbagi A, Guler N. Capecitabine-induced severe hypertriglyceridemia: report of two cases. Ann Pharmacother. 2006; 40: 328-31.

7. Stathopoulos GP, Stergiou GS, Perrea-Kostarelis DN, Dontas IA, Karamanos BG, Karayiannacos PE. Influence of 5-fluorouracil on serum lipids. Acta Oncol. 1995; 34:253-6. 